\title{
On verbally closed subgroups of free solvable groups
}

\section{A. ROMAN'KOV1, E. I. TIMOSHENKO}

Abstract: We study verbally closed subgroups of free solvable groups. A number of results is proved that give sufficient conditions under whose a verbally closed subgroup is turned to be a retract and so algebraically closed of the full group.

\section{INTRODUCTION}

Algebraically closed objects play an important role in the modern algebra. In this paper we study verbally and $l$-verbally closed subgroups of free solvable groups. We establish a connection of them with retracts and algebraically closed subgroups.

Let $\mathcal{K}$ be a class of algebraic structures of language $\mathbf{L}$. A structure $A \in \mathcal{K}$ is said to be algebraically closed in $\mathcal{K}$ if and only if for any positive $\exists$-sentence $\varphi\left(x_{1}, \ldots, x_{n}\right)$ in $\mathbf{L}$ with constants in $A$, if $\varphi\left(x_{1}, \ldots, x_{n}\right)$ is true in a structure $B \in \mathcal{K}$, that has $A$ as a substructure, then $\varphi\left(x_{1}, \ldots, x_{n}\right)$ is true in $A$. See for instances, [1], [2].

An interesting specific case arises when we take as $\mathcal{K}$ the class $\operatorname{sub}(B)$ of all substructures of a structure $B$. Moreover, we can take as $\mathcal{K}$ a pair $\{A, B\}$, where $A$ is a substructure of $B$. Then we can say about algebraic closeness of $A$ in $B$.

Now let $\mathbf{L}$ be the group-theoretical language and $\mathcal{K}$ be a class of groups. In this case, the notion above can be presented in pure group-theoretical terminology. For groups $H$ and $G$ we write $H \leq G$, if $H$ is a subgroup of $G$, and we call $G$ extension of $H$. By an equation $w\left(x_{1}, \ldots, x_{n} ; H\right)=h, h \in H$, in variables (unknowns) $x_{1}, \ldots, x_{n}$ with constants in $H$ we mean an expression in which $w\left(x_{1}, \ldots, x_{n} ; H\right)$ is a group word in $x_{1}, \ldots, x_{n}$ and constants in $H$. We say that an equation $w\left(x_{1}, \ldots, x_{n} ; H\right)=h$ has a solution (is solvable) in $H$ (or more generally, in an extension $G \geq H$ ) if there is a tuple of elements $g_{1}, \ldots, g_{n}$ of $H$ (or more generally of $G$ ) if after a substitution them instead of $x_{1}, \ldots, x_{n}$, respectively, we have the true equality $w\left(g_{1}, \ldots, g_{n} ; H\right)=h$. An equation of the form $w\left(x_{1}, \ldots, x_{n}\right)=h$, in which the left-hand side does not include constants, but only variables, is called, split equation.

In a natural way we define a system of equation and a solution of it.

A notion of an algebraically closed subgroup of a group is rewritten as follows.

\footnotetext{
${ }^{1}$ This research is supported by program of fundamental investigations of SB RAS: I. 1.1.4, project 03142019-0004

${ }^{2}$ This research is supported by RFBR, project18-01-00100a
} 
Definition 1. Let $G$ be a group. A subgroup $H \leq G$ is called algebraically closed in $G$, if any finite system of equations with constants in $H$ that has a solution in $G$ has a solution in $H$.

In [3], the following definition was given.

Definition 2. Let $G$ be a group. A subgroup $H \leq G$ is called verbally closed in $G$, if for any group word $w\left(x_{1}, \ldots, x_{n}\right)$ in variables $x_{1}, \ldots, x_{n}$ without constants and each $h \in H$ a split equation

$$
w\left(x_{1}, \ldots, x_{n}\right)=h,
$$

that has a solution in $G$, is solvable in $H$.

This notion, more weak than algebraic closeness, meanwhile corresponds to much more strong notion of retract.

Definition 3. Retract of a group $G$ is a subgroup $H \leq G$ such that there is an endomorphism $\rho: G \rightarrow H$ that is identical on $H$. Such an endomorphism $\rho$ is called retraction.

Let $F_{r}$ be a free group of rank $r$. Obviously, each free factor of $F_{r}$ is retract. For $r \geq 2, F_{r}$ admits retracts that are not free factors. For example, any element of the form $g=f_{1} u$ of $F_{r}$ with basis $\left\{f_{1}, \ldots, f_{r}\right\}$ where $u$ belongs to the normal closure of $f_{2}, \ldots, f_{r}$, generates a cyclic retract $R$. In this case, a retraction $\rho: F_{r} \rightarrow R$, is defined by map $f_{1} \mapsto g, f_{j} \mapsto 1$ for $j=2, \ldots, r$, but a cyclic group generated by $g$, is a free factor of $F_{r}$ if and only if $g$ is primitive, i.e., it can be included in some basis of $F_{r}$. If $r=2$ and $u$ lies in the commutant of $F_{2}$, then $g=f_{1} u$ is primitive if and only if it conjugates to $f_{1}$. This assertion is followed from a classical Nielsen's result, that every automorphism of $F_{2}$ identical modulo commutant is inner. Element $g=f_{1} f_{2}^{-1} f_{1}^{-1} f_{2} f_{1}$ of $F_{2}$ generates retract but not a free factor.

Remark 1. If $H$ is a retract of $G$ then $H$ is algebraically closed in $G$. Indeed, to obtain a solution in $H$ of an equation $w\left(x_{1}, \ldots, x_{n} ; H\right)=h$ from a solution $x_{i}=g_{i}(i=1, \ldots, n)$ in $G$, it is sufficient to apply a retraction $\rho: G \rightarrow H$. Then $w\left(g_{1}, \ldots, g_{n}, H\right)=h$ implies $\rho\left(w\left(g_{1}, \ldots, g_{n}\right) ; \rho(H)\right)=w\left(\rho\left(g_{1}\right), \ldots, \rho\left(g_{n}\right) ; H\right)=$ $\rho(h)=h$. Obviously that any algebraically closed subgroup $H$ of $G$ is verbally closed in $G$. At the same time, among these three concepts there are no two equivalent one to other. In thesis [11], there are examples of algebraically closed subgroups that are not retracts, as well as examples of verbally closed subgroups that are not algebraically closed. 
In [4], the author proves that intersection of a finite set of retracts of a free group $F_{r}$ is retract. In [5], there is a question 17.19 (the same as the question F11 in [6]): If $H$ is a finitely generated subgroup of $F_{r}$ and $R$ is a retract of $F_{r}$, is it followed that the intersection $R \cap H$ is retract of $H$ ? Recently this question was answered negatively in [7].

Thus the concept of verbal closeness is weaker than algebraic closeness. However in [3] the authors proved that any verbally closed subgroup $H$ of a free group $F_{r}(r \in \mathbb{N})$ is a retract of $F_{r}$. It follows that the sets of verbally closed subgroups, algebraically closed subgroups and retracts of $F_{r}$ coincide.

In [8], a similar statement was shown for free nilpotent groups. Let $N_{r, c}$ denote a free nilpotent group of a finite rank $r$ and nilpotency class $c$. Then the following properties of a subgroup $H \leq N_{r, c}$ are equivalent one to other: verbal closeness, algebraic closeness, the property "to be a retract" and the property "to be a free factor" with respect to the variety $\mathfrak{N}_{c}$ of all nilpotent groups of nilpotency class $\leq c$.

In the series of papers [9], [10], [11], [12] and [13] verbal closeness was studied. The following property was introduced. A group $H$ is a strongly verbally closed if $H$ is an algebraically closed subgroup in any group containing $H$ as a verbally closed subgroup. The number of results was presented showing that many groups are strongly verbally closed.

Now we introduce the following new notion.

Definition 4. Let $G$ be a group. For any $l \in \mathbb{N}$, a subgroup $H \leq G$ is said to be $l$-verbally closed in $G$ if every system of $l$ split equations on any $n$ variables

$$
w_{i}\left(x_{1}, \ldots, x_{n}\right)=h_{i}, h_{i} \in H, i=1, \ldots, l,
$$

that has a solution in $G$, is solvable in $H$.

In the case $l=1$ this notion means verbal closeness.

Remark 2. Note that the verbal closeness does not imply $l$-verbal closeness of $H$ in $G$. Indeed, every equation $w\left(x_{1}, \ldots, x_{n} ; H\right)=h, h \in H$, is equivalent to a system of split equations with right sides in $H$. This system can be obtained from the initial equation if we change each coefficient $h$ to new variable $x_{h}$ and add the corresponding split equation $x_{h}=h$ to the system. Hence any nonalgebraically closed subgroup $H \leq G$ is non-l-verbally closed for some $l$. We noted in Remark 1, that there are verbally closed but non-algebraically closed subgroups of some groups.

Further in the paper we denote a conjugate by $g^{f}=f g f^{-1}$, and commutator by $[f, g]=f g f^{-1} g^{-1}$. Here $f, g$ are elements of arbitrary group $G$. By $G^{\prime}$ we 
denote the derived subgroup (commutant) of $G$. By $\gamma_{i} G$ we mean the $i$-th member of the low central series in $G$ (in particular, $\gamma_{1} G=G, \gamma_{2} G=G^{\prime}$ ). By $G^{(i)}$ we denote the $i$-th member of the derived series in $G$ (in particular, $\left.G^{(0)}=G, G^{(1)}=G^{\prime}\right)$. By $\left\langle g_{1}, \ldots, g_{k}\right\rangle$ we denote the subgroup generated by elements $g_{1}, \ldots, g_{k}$ in $G$. The variety of all abelian grous is denoted as $\mathfrak{A}$, the variety of all nilpotent groups of nilpotency class $c(c \in \mathbb{N})$ is denoted as $\mathfrak{N}_{c}$. By $\mathfrak{A}^{d}(d \in \mathbb{N})$ we mean the variety of all solvable groups of the solvability class $\leq d$.

For any tuple of varieties $\mathfrak{B}_{i}, i=1, \ldots, k, \prod_{i=1}^{k} \mathfrak{B}_{i}$ denotes their product. We write $F_{r}(\mathfrak{B})$ to denote a free group of rank $r$ in $\mathfrak{B}$. Further in the paper we assume that any rank $r$ is finite. For some relatively free groups we use specific denotions: $A_{r}=F_{r}(\mathfrak{A}), N_{r, c}=F_{r}\left(\mathfrak{N}_{r, c}\right), S_{r, d}=F_{r}\left(\mathfrak{A}^{d}\right)$.

Let $H$ be a finitely generated subgroup of a group $G$. Then $r_{a b}(H)$ denotes a rank, i.e., the minimal number of generators of $H G^{\prime} / G^{\prime}$.

If $H$ is verbally closed then $G \cap G^{\prime}=H^{\prime}$. Indeed, if $h \in G^{\prime} \cap H$, then some equation of the form $h=\prod_{j=1}^{t}\left[x_{j}, x_{j}^{\prime}\right]$ is solvable in $G$. Then this equation has a solution in $H$, i.e., $h \in H^{\prime}$. Then $H / H^{\prime} \cong H / H \cap G^{\prime} \cong H G^{\prime} / G^{\prime}$.

The following assertion is followed from the statements above.

Proposition 1. Let $G=\left\langle g_{1}, \ldots, g_{k}, \ldots\right\rangle$ be a solvable group and $H$ be a subgroup of $G$ such that $r_{a b}(H)=0$, i.e., $H \leq G^{\prime}$. If $H$ is verbally closed in $G$, then $H=1$.

Proof. Suppose that $h \in H, h \neq 1$. Let $h \in G^{(n)} \backslash G^{(n+1)}, n \geq 1$. Then $h$ can be expressed in the form $h=\prod_{i=1}^{m} h_{i}$, where every factor $h_{i}$ is written as $\left[v_{i}, w_{i}\right], v_{i}, w_{i} \in G^{(n-1)}$. Let $v_{i}=v_{i}\left(g_{1}, \ldots, g_{r}\right)$ and $w_{i}=$ $w_{i}\left(g_{1}, \ldots, g_{r}\right)$ are the corresponding expressions of the elements $v_{i}, w_{i}$ in generators of $G$. The words $v_{i}\left(x_{1}, \ldots, x_{n}\right)$ and $w_{i}\left(x_{1}, \ldots, x_{n}\right)$ are chosen in $F_{n}^{(n-1)}$, where $F_{n}$ is the free group with basis $\left\{x_{1}, \ldots, x_{n}\right\}$. Hence, the equation $\prod_{i=1}^{m}\left[v_{i}\left(x_{1}, \ldots, x_{r}\right), w_{i}\left(x_{1}, \ldots, x_{r}\right)\right]=h$ has a solution in $G$. Obviously this equation has no solutions in $G^{\prime}$ because any substitution of elements from $G^{\prime}$ instead of variables gives an element in $G^{(n+1)}$. Especially, this equation is not solvable in $H$.

The following statements are main results of this paper.

Theorem 1. Let $H$ be a finitely generated verbally closed subgroup of a free solvable group $S_{r, d}$. If $r_{a b}(H)=1$, then $H$ is a retract.

Theorem 2. Let $H$ be a finitely generated verbally closed subgroup of a free solvable group $S_{r, d}$. If $r_{a b}(H)=r$, then $H=S_{r, d}$. 
Theorem 3. Every 2-generated verbally closed subgroup $H$ of a free solvable group $S_{r, d}$ is a retract.

Theorem 4. For any $l \in \mathbb{N}$, every finitely generated l-verbally closed subgroup $H$ of a free metabelian group $M_{r}$ such that $r_{a b}(H)=l+1$ is a retract.

\section{§1. PRELIMINARY RESULTS}

Recall that a set of elements $\left\{g_{1}, \ldots, g_{n}\right\}$ of a group $G$ is test set if any endomorphism $\varphi: G \rightarrow G$, that fixes every element $g_{i}(i=1, \ldots, n)$, is an automorphism of $G$. An element $g \in G$ is said to be test element if $\{g\}$ is test set. Test rank $\operatorname{tr}(G)$ is defined as the minimal number of elements in a test set of $G$. See [14, [15], [16], [17], [18] for details and results about test sets and test ranks of groups.

Let $F_{r}$ be a free group of rank $r$ and let $V$ be a normal subgroup of $F_{r}$. A test set $\left\{g_{1}, \ldots, g_{n}\right\} \subseteq G=F_{r} / V^{\prime}$ is said to be strongly test set if any endomorphism $\varphi: G \rightarrow G$ fixing every element $g_{i}$ is inner automorphism that acts as conjugate by element from $V / V^{\prime}$.

Recall, that a variety of the form $\mathfrak{N}_{c_{1}} \ldots \mathfrak{N}_{c_{m}}, m \geq 1$ is called polynilpotent of class $\bar{c}=\left(c_{1}, \ldots, c_{m}\right)$. We consider a polynilpotent variety of the form $\mathrm{P} \mathfrak{N}_{\bar{c}}=$ $\mathfrak{A N}_{c_{1}} \ldots \mathfrak{N}_{c_{m}}, m \geq 1$, of class $\bar{c}=\left(2, c_{1}, \ldots, c_{m}\right)$. Note that class $\bar{c}=(2,2, \ldots, 2)$ corresponds to $\mathfrak{A}^{m+1}$. The following lemma follows from results and their proofs of the papers ([16], [17]) by C.K. Gupta and the second author.

Lemma 1. A relatively free group $F_{r}\left(P \mathfrak{N}_{\bar{c}}\right)$ of rank $r \geq 2$ of the variety $P \mathfrak{N}_{\bar{c}}, m \geq 1$, admits a strongly test set consisting of $(r-1)$ elements.

Proof. Let $F_{r} / V$ be a free group of rank $r$ of the polynilpotent variety $\mathfrak{N}_{c_{1}} \ldots \mathfrak{N}_{c_{m}}, m \geq 1$. The main statement in [16] is computing of the test rank of any free solvable group. In fact, in this paper a test rank of $F_{r} / V^{\prime}$ was compute. Especially, it was shown that the test rank of a free group of the polynilpotent variety of the form $\mathfrak{A N}_{c_{1}} \ldots \mathfrak{N}_{c_{m}}$ was computed. Moreover, it was shown how a test set $\left\{g_{1}, \ldots, g_{r-1}\right\}$ for $F_{r} / V^{\prime}$ can be constructed such that: if an endomorphism $\varphi$ fixes every element of this set then $\varphi$ is an inner automorphism that induces identity map on $V / V^{\prime}$. In [17], it was shown that the mentioned above inner automorphism is induced by conjugate to an element that lies in $V / V^{\prime}$, then $\left\{g_{1}, \ldots, g_{r-1}\right\}$ is strong test set. 
Lemma 2. Let $G=F_{r} / V^{\prime}=F_{r}\left(\mathfrak{A N}_{c_{1}} \ldots \mathfrak{N}_{c_{m}}\right), m \geq 1, r \geq 2$, and let $\left\{z_{1}, \ldots, z_{r}\right\}$ be a basis of $G$. Let $H \leq G$ is isomorphic to $F_{n}\left(\mathfrak{A N}_{c_{1}} \ldots \mathfrak{N}_{c_{m}}\right)$ for some $n \geq 2$. Let $T=\left\{t_{1}\left(z_{1}, \ldots, z_{r}\right), \ldots, t_{n-1}\left(z_{1}, \ldots, z_{r}\right)\right\}$ be a strong test set in H. Suppose that

$$
t_{i}\left(h_{1}, \ldots, h_{r}\right)=t_{i}\left(z_{1}, \ldots, z_{r}\right), \quad i=1, \ldots, n-1,
$$

for some elements $h_{1}, \ldots, h_{r} \in H$. Then map

$$
z_{j} \mapsto h_{j}, j=1, \ldots, r
$$

is extended to a retraction $G \rightarrow H$.

Proof. Extend map $z_{j} \mapsto h_{j}, j=1, \ldots, r$, to endomorphism $\varphi: G \rightarrow H$. Let $\left.\varphi\right|_{H}$ be restriction of $\varphi$ to $H$, that is an endomorphism of $H$.

Then $\left.\varphi\right|_{H}\left(t_{i}\left(z_{1}, \ldots, z_{r}\right)\right)=\varphi\left(t_{i}\left(z_{1}, \ldots, z_{r}\right)\right)=t_{i}\left(\varphi\left(z_{1}\right), \ldots, \varphi\left(z_{r}\right)\right)=$ $t_{i}\left(h_{1}, \ldots, h_{r}\right)=t_{i}\left(z_{1}, \ldots, z_{r}\right), i=1, \ldots, n-1$. Hence $\left.\varphi\right|_{H}$ is an inner automorphism $\alpha_{a}$ of $H$ induced by $a \in V / V^{\prime}$. Consider an inner automorphism $\psi=\varphi_{a^{-1}} \varphi$, where $\varphi_{a^{-1}}$ of $G$ corresponds to $a^{-1}$. For $h \in H$ we have

$$
\psi(h)=\varphi_{a^{-1}} \varphi(h)=\varphi_{a^{-1}}\left(h^{a}\right)=h,
$$

and for $g \in G$

$$
\psi(g)=(\varphi(g))^{a^{-1}} \in H .
$$

Thus $\psi$ is a seeking retraction onto $H$.

The lemmas above imply the following assertion.

Proposition 2. Let $H$ be a subgroup of $F_{r}\left(\mathfrak{A N}_{c_{1}} \ldots \mathfrak{N}_{c_{l}}\right)$ generated by $(m+1)$ elements and rank of $H_{a b}=H / H^{\prime}$ is equal to $m+1$. Then, if $H$ is m-verbally closed subgroup, it is a retract.

Lemma 3. ([8]). Let $N$ be a verbal subgroup of a group $G$. If $H$ is a verbally closed subgroup of $G$, then its image $H_{N}=H N / N$ is verbally closed in $G_{N}=$ $G / N$. Specifically, if $G / G^{\prime}$ is a free abelian group of a finite rank then the image $\bar{H}$ of its verbally closed subgroup $H$ in $G / G^{\prime}$ is a direct factor.

Lemma 4. Let $G=S_{r, d}$ be a free solvable group of class $d \geq 2$ with basis $z_{1}, \ldots, z_{r}, r \geq 2$. Let $H \leq G$ be a verbally closed subgroup generated by elements $c_{1} z_{1}, \ldots, c_{r} z_{r}, c_{r+1} \ldots$, where any $c_{i}$ lies in $G^{(d-1)}$. Then $H^{(d-1)}$ is $\mathbb{Z}\left[H / H^{(d-1)}\right]$-closed subgroup of $G^{(d-1)}$, i.e., if for any $c \in G^{(d-1)}$ and $0 \neq \alpha \in \mathbb{Z}\left[G / G^{(d-1)}\right]$ we have $c^{\alpha} \in H^{(d-1)}$, then $c \in H^{(d-1)}$. 
Proof. Denote $A=G / G^{(d-1)}, B=H / H^{(d-1)}$, then $G^{(d-1)} \cap H=H^{(d-1)}$.

Let $1 \neq c^{\alpha} \in H^{(d-1)}$. Write $c$ as $c=c\left(z_{1}, \ldots, z_{r}\right)$. Then $\alpha$ has an expression of the form $\alpha=\Sigma m_{p} \bar{h}_{p}$, where $m_{p} \in \mathbb{Z}, \bar{h}_{p} \in B$. Let $a_{i}=z_{i} G^{(d-1)}$. Then every element $\bar{h}_{p}$ lies in the subgroup generated by $a_{1}, \ldots, a_{r}$, i.e., $\alpha=\alpha\left(a_{1}, \ldots, a_{r}\right)$.

Consider an equation in unknowns $x_{1}, \ldots, x_{r}$ :

$$
\begin{aligned}
& c\left(x_{1}, \ldots, x_{r}\right)^{\left(1-\bar{x}_{1}^{m_{1}}\right)\left(1-\bar{x}_{1}^{-m_{1}}\right) \ldots\left(1-\bar{x}_{r}^{m_{r}}\right)\left(1-\bar{x}_{r}^{m_{r}}\right) \alpha\left(\bar{x}_{1}, \ldots, \bar{x}_{r}\right)} \\
= & c\left(z_{1}, \ldots, z_{r}\right)^{\left(1-a_{1}^{m_{1}}\right)\left(1-a_{1}^{-m_{1}}\right) \ldots\left(1-a_{r}^{m_{r}}\right)\left(1-a_{r}^{-m_{n}}\right) \alpha\left(a_{1}, \ldots, a_{r}\right),}
\end{aligned}
$$

where $m_{i}$ are integers. This equation has a solution in $G$. Hence, it has a solution $x_{i}=h_{i}$ in $H$. Then

$$
\begin{aligned}
& c\left(h_{1}, \ldots, h_{r}\right)^{\left(1-\bar{h}_{1}^{m_{1}}\right)\left(1-\bar{h}_{1}^{-m_{1}}\right) \ldots\left(1-\bar{h}_{r}^{m_{r}}\right)\left(1-\bar{h}_{r}^{-m_{r}}\right) \alpha\left(\bar{h}_{1}, \ldots, \bar{h}_{r}\right)}= \\
& =c\left(z_{1}, \ldots, z_{r}\right)^{\left(1-a_{1}^{m_{1}}\right)\left(1-a_{1}^{-m_{1}}\right) \ldots\left(1-a_{r}^{m_{r}}\right)\left(1-a_{r}^{-m_{r}}\right) \alpha\left(a_{1}, \ldots, a_{r}\right) .}
\end{aligned}
$$

Here $\bar{g}$ denotes the image of $g \in G$ in $A$.

The group ring $\mathbb{Z}[G]$ is equipped by the left Fox derivations $\partial_{j}(g)$ with values in $\mathbb{Z}[A]$ (see details in [18, [19], [20], [21]). For $\alpha \in \mathbb{Z}[A]$ and $b \in G^{(d-1)}$ the following equality is valid:

$$
\partial_{i}\left(b^{\alpha}\right)=\alpha \partial_{i}(b)
$$

Computing the values of $j$ th derivation of two sides of the equality (2) we obtain

$$
\begin{gathered}
\left(1-\bar{h}_{1}^{m_{1}}\right)\left(1-\bar{h}_{1}^{-m_{1}}\right) \ldots\left(1-\bar{h}_{r}^{m_{r}}\right)\left(1-\bar{h}_{r}^{-m_{r}}\right) \alpha\left(\bar{h}_{1}, \ldots, \bar{h}_{r}\right) \partial_{j}\left(c\left(h_{1}, \ldots, h_{r}\right)\right)= \\
=\left(1-a_{1}^{m_{1}}\right)\left(1-a_{1}^{-m_{1}}\right) \ldots\left(1-a_{r}^{m_{r}}\right)\left(1-a_{n}^{-m_{r}}\right) \alpha\left(a_{1}, \ldots, a_{r}\right) \partial_{j}(c) .
\end{gathered}
$$

Let $\Delta$ be the fundamental ideal of $\mathbb{Z}[A] . \Delta$ is endowed with a norm $\omega$ (see [27]). Namely, for an element $u \in \Delta$ we set $\omega(u)=n$, where $u \in \Delta^{n} \backslash \Delta^{n+1}$.

We'll show that no $h_{i}$ lies in $G^{\prime}$. Since $c=c\left(z_{1}, \ldots, z_{r}\right) \neq 1$, there is $j$ for which $\partial_{j}(c) \neq 0$. We choose $j$, for which the norm of $\partial_{j}(c)$ is minimal among all $\omega\left(\partial_{1}(c)\right), \ldots, \omega\left(\partial_{r}(c)\right)$. We can assume for simplicity that $j=1$. In (3) we compare the norms of the left and the right sides. By the known rule we have

$$
\partial_{1}\left(c\left(h_{1}, \ldots, h_{r}\right)\right)=\partial_{1}(c)\left[\bar{h}_{1}, \ldots, \bar{h}_{r}\right] \partial_{1}\left(h_{1}\right)+\ldots+\partial_{r}(c)\left[\bar{h}_{1}, \ldots, \bar{h}_{r}\right] \partial_{1}\left(h_{r}\right),
$$

where $\partial_{i}(c)\left[\bar{h}_{1}, \ldots, \bar{h}_{r}\right]$ means a result of substitution to $\partial_{i}(c)$ of elements $\bar{h}_{q}$ instead $\bar{z}_{q}$. By choice of $j$ we obtain $\omega\left(\partial_{1} c\left(h_{1}, \ldots, h_{r}\right)\right) \leq \omega\left(\partial_{1}(c)\right)$. If at least one element $h_{i}$ belongs to $G^{\prime}$, the norm of the left side of (3) is less than the norm of the right side, because $\omega\left(1-a_{i}\right)=1<\omega\left(1-\bar{h}_{i}\right)$, for $h_{i} \in G^{\prime}$.

In [16], the following statement was proved. Let $S$ be a free polynilpotent group of rank $r \geq 2$ with basis $\left\{s_{1}, \ldots, s_{r}\right\}$, and $0 \neq \alpha \in \mathbb{Z}[S]$. Let $p_{i}$ be the least 
$s_{i}$ - exponent of $\alpha$, and $q_{i}$ be the greatest $s_{i}$ - exponent of it. Let a positive integer $m$ is greater than

$$
\sum_{i=1}^{r}\left(\left|p_{i}\right|+\left|q_{i}\right|\right) .
$$

For $y_{1} \in G \backslash S^{\prime}$, equality

$$
\left(1-s_{1}^{m}\right) \alpha=\left(1-y_{1}^{m}\right) \beta
$$

is possible for $\beta \in \mathbb{Z}[S]$ if and only if $y_{1}=s_{1}$ or $y_{1}=s_{1}^{-1}$.

Now we choose an available $m_{i}$. Then $\bar{h}_{1}=a_{1}^{ \pm 1}$. The ring $\mathbb{Z}[B]$ has no zero divisors. The expression $\left(1-a_{i}^{m_{i}}\right)\left(1-a_{i}^{-m_{i}}\right)$ is not changed under substitution $a_{i}$ to $a_{i}^{-1}$. We cancel step by step (3) to $\left(1-a_{i}^{m_{1}}\right)\left(1-a_{i}^{-m_{1}}\right)$ and obtain $\bar{h}_{i}=a_{i}^{ \pm 1}$ for all $i=1, \ldots, r$. Then we get $\partial_{i}\left(c\left(h_{1}, \ldots, h_{r}\right)\right)=\partial_{i}(c)$, and

$$
c=c\left(h_{1}, \ldots, h_{r}\right) \in H^{(d-1)} .
$$

Lemma 5. Let $H=\langle h\rangle$ be a non-trivial cyclic subgroup of $G=S_{r, d}$. Then the following conditions are equivalent:

1. $H$ is a verbally closed in $G$;

2. $H$ is a retract of $G$;

3. the image $\bar{h}$ of $h$ in $G / G^{\prime}$, i.e., in the free abelian group $A_{r}$, is primitive. Proof. By Lemma 31 implies 3.

Let we show that 3 implies 2. Write $h$ as $h=a u$, where $a$ is a primitive element of $A_{r}, u \in G^{\prime}$. Since any basis of $A_{r}$ is induced by a basis of $G$ (moreover, it is induced by a basis of $F_{r}$, see for example [21]). Hence there is a preimage $z$ of $a$ in $G$ that belongs to a basis of $G$. We take the endomorphism of $G$ that maps $z$ to, and any other element of this basis to 1 . We see that this endomorphism is a retraction $G \rightarrow H$.

By Remark 1, 2 implies 1.

Lemma 6. Let $H=\langle g, f\rangle$ be a 2-generated non-cyclic subgroup of a free solvable group $G=S_{r, d}$ of rank $r \geq 2$. Then the following statement is valid: if $H$ is a verbally closed subgroup of $G$, then $g, f$ induce in $A_{r}=G / G^{\prime}$ (free abelian group of rank $r$ ) a direct factor of rank 2 , and $H$ is a free solvable group of rank 2 and class $d$. 
Proof. By Lemma 3, the images of $g, f$ in $A_{r}$ generate a verbally closed subgroup, that is a direct factor of full group. It remains to prove, that this factor is non-cyclic. By Lemma 3 and induction on $d$ we assume that the image of $H$ in $S=G / G^{(d-1)}$ is generated by the image $\bar{g}$ of $g$, and $f$ lies in $G^{(d-1)}$. Let $f\left(z_{1}, \ldots, z_{r}\right)$ be an expression of $f$ as a word in generators of $G$. Equation $f\left(x_{1}, \ldots, x_{r}\right)=f$ is solvable in $G$. Hence, it has a solution $h_{1}, \ldots, h_{r}$ in $H$. The components of this solution are written as $h_{i}=g^{t_{i}} f^{\alpha_{i}}, t_{i} \in \mathbb{Z}$, where $\alpha_{i} \in \mathbb{Z}[\bar{g}], i=1, \ldots, r$. Then $f\left(h_{1}, \ldots, h_{r}\right)=f\left(g^{t_{1}}, \ldots, g^{t_{r}}\right) f^{\delta}$, where $\delta \in \mathbb{Z}[\bar{g}]$ is easily to compute by collecting exponents of $f$ in $f\left(h_{1}, \ldots, h_{r}\right)$. It remains to note that $\delta$ lies in the fundamental ideal of $\mathbb{Z}[\bar{g}]$, because $f\left(x_{1}, \ldots, x_{r}\right)$ is a commutator word. Then $f^{1-\delta}=1$ for $1-\delta \neq 0$ that is impossible in view of well-known fact that $G^{(d-1)}$ has no module torsion (see for example [21]). By Baumslag's theorem [22] $H=\langle g, f\rangle$ is a free solvable group of rank $r$ and class $d$ with basis consisting of the elements described above.

The following statement was proved in [23] (see also [21])

Lemma 7. ([23]). Let $M_{2}$ be the free metabelian group of rank 2 with basis $\left\{z_{1}, z_{2}\right\}$. Let $x_{1}=g_{1}, x_{2}=g_{2}$ be a solution of

$$
\left[x_{1}, x_{2}, x_{2}, x_{1}\right] \equiv\left[z_{1}, z_{2}, z_{2}, z_{1}\right] .
$$

Then $g_{i} \equiv z_{i}^{\varepsilon_{i}}\left(\bmod M_{2}^{\prime}\right), \varepsilon_{i} \in\{ \pm 1\}$.

Here and further $\left[g_{1}, g_{2}, \ldots, g_{k}\right]$ means a simple commutator that is defined inductively: $\left[g_{1}, g_{2}, \ldots, g_{k}\right]=\left[\left[g_{1}, g_{2}, \ldots, g_{k-1}\right], g_{k}\right]$.

\section{§2. PROOFS OF THE MAIN RESULTS}

Proof of Theorem 1. Denote $G=S_{r, d}$. We use induction on $d$. The case $d=1$ is trivial. Suppose that $r, d \geq 2$. By induction and Lemma 3 we can choose a basis $\left\{z_{1}, \ldots, z_{r}\right\}$ of $G$ in such way that

$$
H=\left\langle z_{1} c_{1}, c_{2}, \ldots, c_{m}\right\rangle,
$$

where $c_{1}, \ldots, c_{m} \in G^{(d-1)}$. The quotient $G / G^{(d-1)}$ is isomorphic to $S_{r, d-1}$. Denote $C=G^{(d-1)}$ and $A=G / C$. Let $\left\{a_{1}, \ldots, a_{r}\right\}$ be a basis of $A$ corresponding to $\left\{z_{1}, \ldots, z_{r}\right\}$.

We'll show that each $c_{i}$ is equal to 1 . We write any $c_{i}$ in the form $c_{i}=$ $c_{i}\left(z_{1}, \ldots, z_{r}\right)$, where $c_{i}\left(x_{1}, x_{2}, \ldots, x_{r}\right)$ is a word that lies in the $(d-1)$ th member 
of the derived series of the free group $F\left(X_{r}\right), X_{r}=\left\{x_{1}, \ldots, x_{r}\right\}$. Since $H$ is verbally closed there exist elements $h_{1}, \ldots, h_{r} \in H$, such that

$$
c_{i}=c_{i}\left(h_{1}, \ldots, h_{r}\right) .
$$

From (5) we get the equalities:

$$
c_{i}=c_{1}^{-\delta_{i 1}} \ldots c_{i}^{-\delta_{i i}} \ldots c_{m}^{-\delta_{i m}}, i=1, \ldots, m,
$$

where $\delta_{i j}\left(a_{1}\right) \in \mathbb{Z}\left[a_{1}^{ \pm 1}\right]$. Let $\nu_{i}=1+\delta_{i i}$. Each element $\delta_{i j}$ for $i \neq j$ lies in ideal $i d\left(a_{1}-1\right)$ of $\mathbb{Z}\left[a_{1}^{ \pm 1}\right]$, and any $\nu_{i}=1$ modulo this ideal.

Then (6) implies a system of equalities:

$$
\begin{gathered}
c_{1}^{\nu_{1}} c_{2}^{\delta_{12}} \ldots c_{m}^{\delta_{1 m}}=1, \\
c_{1}^{\delta_{21}} c_{2}^{\nu_{2}} \ldots c_{m}^{\delta_{2 m}}=1, \\
\ldots \ldots \ldots \ldots \\
c_{1}^{\delta_{m 1}} c_{2}^{\delta_{m 2}} \ldots c_{m}^{\nu_{m}}=1 .
\end{gathered}
$$

Compute Fox derivations of the left sides that are equal to 0 .

$$
\begin{gathered}
\nu_{1} \partial_{j}\left(c_{1}\right)+\delta_{12} \partial_{j}\left(c_{2}\right)+\ldots+\delta_{1 m} \partial_{j}\left(c_{m}\right)=0 \\
\delta_{21} \partial_{j}\left(c_{1}\right)+\nu_{2} \partial_{j}\left(c_{2}\right)+\ldots+\delta_{2 m} \partial_{j}\left(c_{m}\right)=0 \\
\ldots \ldots \ldots \\
\delta_{m 1} \partial_{j}\left(c_{1}\right)+\delta_{m 2} \partial_{j}\left(c_{2}\right)+\ldots+\nu_{m} \partial_{j}\left(c_{m}\right)=0
\end{gathered}
$$

where $j=1, \ldots, m$. Determinant of each system (7) is 1 modulo $i d\left(a_{1}-1\right)$, thus it is not 0 . Hence the system has the only zero solution. It follows that each $c_{i}$ is 1 . By Lemma 5 we finish the proof.

Proof of Theorem 2). Denote $G=S_{r, d}$. We use induction on $d$. The case $d=1$ is trivial. We assume that $r, d \geq 2$. By induction and Lemma 3 we construct a basis $\left\{z_{1}, \ldots, z_{r}\right\}$ of $G$ such that

$$
H=\left\langle z_{1} c_{1}, \ldots, z_{r} c_{r}, c_{r+1}, \ldots, c_{m}\right\rangle,
$$

where $c_{1}, \ldots, c_{r}, c_{r+1}, \ldots, c_{m} \in C$. It is enough to check that each element $c \in C$ lies $H^{(n-1)}$.

Denote $C=G^{(d-1)}, A=G / C \simeq S_{r, d-1}$. Abelian group $C$ is a right $\mathbb{Z}[A]$-module in which action of $a \in A$ to $c \in C$ is defined as $a^{-1} c a$. Elements 
$a_{i}=z_{i} C, i=1, \ldots, r$, give a basis of $A$. Let $T$ be a free right $\mathbb{Z}[A]$-module with basis $\left\{t_{1}, \ldots, t_{r}\right\}$. We take the Magnus embedding of $G$ into split extension

$$
M=\left(\begin{array}{cc}
A & 0 \\
T & 1
\end{array}\right) .
$$

See details in [18], [21]. Any element $z_{i}$ maps to

$$
\left(\begin{array}{cc}
a_{i} & 0 \\
t_{i} & 1
\end{array}\right)
$$

The image of $C$ in $M$ is a submodule of $T$, that is identified with $C$. An element $t_{1} u_{1}+\ldots t_{r} u_{r}, u_{i} \in \mathbb{Z}[A]$, lies in $C$ if and only if

$$
u_{1}\left(a_{1}-1\right)+\ldots+u_{r}\left(a_{r}-1\right)=0 .
$$

The image of $C$ in $M$ is a submodule of $T$, consisting of all elements that satisfy to (8). We keep its denotion $C$.

It is well-known that $\mathbb{Z}[A]$ satisfies to the right Ore condition [26], i.e., for any pair of non-zero elements elements $\alpha, \beta \in \mathbb{Z}[A]$ there are non-zero elements $\mu, \nu \in \mathbb{Z}[A]$ such that $\alpha \mu=\beta \nu \neq 0$. Since $\mathbb{Z}[A]$ is also a domain it is embedded into a sfield of fractions $P$. Then $T$ embeds into a linear space $V$ of dimension $r$ over $P$. By (8) we conclude that the dimension of the linear subspace $C^{P}$ generated by $C$ in $V$ is $r-1$.

Let we consider a subset of non-trivial elements $\left\{w_{i}=w_{i}\left(z_{1}, z_{i}\right), i=\right.$ $2, \ldots, r\}$ of $C$. It is easily compute that the image of $w_{i}$ under Magnus embedding in $M$ (more exactly in $T$ ) has the form $t_{1} \gamma_{1}+t_{i} \gamma_{i}$, where $\gamma_{1}$ and $\gamma_{i}$ are non-zero. Independent of $t_{1} \gamma_{1}+t_{i} \gamma_{i}, i=2, \ldots, r$ in $T$ is clear. We are to check that elements $w_{i}\left(z_{1} c_{1}, z_{i} c_{i}\right), i=2, \ldots, r$, are linearly independent over $\mathbb{Z}[A]$.

Denote by $G_{1}$ the subgroup generated by elements $z_{1} c_{1}, \ldots, z_{r} c_{r}$. By Baumslag's theorem [22] the map

$$
\varphi: z_{i} \mapsto z_{i} c_{i}, \quad i=1, \ldots, r
$$

extends to isomorphism $G$ and $G_{1}$, and then to isomorphism of $C$ and $G_{1}^{(d-1)}$, and also to isomorphism of $\mathbb{Z}[A]$ and $\mathbb{Z}\left[G_{1} / G_{1}^{(d-1)}\right]$. Moreover, $\mathbb{Z}[A]$-module $C$ is isomorphic to $\mathbb{Z}\left[G_{1}^{(d-1)}\right]$-module $G_{1}^{(d-1)}$. Any of these isomorphisms is denoted by $\varphi$.

We are to check that elements $w_{i}\left(z_{1} c_{1}, z_{i} c_{i}\right), i=2, \ldots, r$ are independent over $\mathbb{Z}[A]$.

Suppose that it is not happen:

$$
w_{2}\left(z_{1} c_{1}, z_{2} c_{2}\right)^{\beta_{2}\left(z_{1}, \ldots, z_{r}\right)} \ldots w_{r}\left(z_{1} c_{1}, z_{r} c_{r}\right)^{\beta_{r}\left(z_{1}, \ldots, z_{r}\right)}=1
$$


for some $\beta_{i} \in \mathbb{Z}[A]$, at least one of them is not 0 . Hence

$$
w_{2}\left(z_{1} c_{1}, z_{2} c_{2}\right)^{\beta_{2}\left(z_{1} c_{1}, \ldots, z_{r} c_{r}\right)} \ldots w_{r}\left(z_{1} c_{1}, z_{r} c_{r}\right)^{\beta_{r}\left(z_{1} c_{1}, \ldots, z_{r} c_{r}\right)}=1 .
$$

We apply $\varphi^{-1}$ and get a contradiction with independence of $w_{2}, \ldots, w_{r}$ over $\mathbb{Z}[A]$.

We are to check that elements $w_{i}\left(z_{1} c_{1}, z_{i} c_{i}\right), i=2, \ldots, r$, are linearly independent not only over $\mathbb{Z}[A]$, but over $P$ too. Elements of $P$ are equivalency classes of $\mathbb{Z}[A] \times \mathbb{Z}[A]$, that usually denoted as $\alpha \beta^{-1}$ for $0 \neq \beta, \alpha \in \mathbb{Z}[A]$. Suppose that there is a non-trivial linear combination over $P$ :

$$
w_{2}\left(z_{1} c_{1}, z_{2} c_{2}\right) \alpha_{2} \delta_{2}^{-1}+\ldots+w_{r}\left(z_{1} c_{1}, z_{r} c_{r}\right) \alpha_{r} \delta_{r}^{-1}=0 .
$$

Since $\mathbb{Z}[A]$ is the Ore domain every a finite set of its elements has a right common factor. Take such factor $\delta$ for $\delta_{2}, \ldots, \delta_{r}$. By definition $0 \neq \delta=\delta_{i} \gamma_{i}, i=$ $2, \ldots, r, \gamma_{i} \in \mathbb{Z}[A]$. Then

$$
w_{2}\left(z_{1} c_{1}, z_{2} c_{2}\right) \alpha_{2} \gamma_{2}+\cdot+w_{r}\left(z_{1} c_{1}, z_{r} c_{r}\right) \alpha_{r} \gamma_{r}=0 .
$$

This contradicts to linear independence of elements $w_{i}\left(z_{1} c_{1}, z_{i} c_{i}\right), i=2, \ldots, r$, over $\mathbb{Z}[A]$.

We proved that elements $w_{2}\left(z_{1} c_{1}, z_{2} c_{2}\right), \ldots, w_{r}\left(z_{1} c_{1}, z_{r} c_{r}\right)$ are linearly independent over $P$.

There are elements $0 \neq \sigma, \sigma_{1}, \ldots, \sigma_{r-1} \in P$ such that

$$
c^{\sigma}=w_{2}\left(z_{1} c_{1}, z_{2} c_{2}\right)^{\sigma_{1}} \ldots w_{r}\left(z_{1} c_{1}, z_{r} c_{r}\right)^{\sigma_{r-1}} .
$$

By multiplying all $\sigma, \sigma_{1}, \ldots, \sigma_{r-1}$ to available element $u \in \mathbb{Z}[A]$, we can assume that $\sigma, \sigma_{1}, \ldots, \sigma_{r-1} \in \mathbb{Z}[A]$. Thus $c^{\sigma} \in H^{(d-1)}$. Lemma 4 implies that $c \in H^{(d-1)}$.

Proof of Theorem 3. By Lemma $6 H$ is solvable of rank 2. By Baumslag's theorem [22] it isomorphic to $S_{2 d}$. By Lemma1 $H$ contains a strong test element. By Lemma $2 H$ is a retract.

Proof of Theorem 4. Denote $G=M_{r}$. Let $\left\{z_{1}, \ldots, z_{r}\right\}$ be a basis of $G$, and let $\left\{a_{1}, \ldots, a_{r}\right\}$ be the corresponding basis of $A_{r}=G / G^{\prime}$.

By Lemma 3 we can assume that $H=\left\langle h_{1}, \ldots, h_{l+1}, h_{l+2}, \ldots h_{l+t}\right\rangle$, where $h_{i} \equiv$ $z_{i}\left(\bmod G^{\prime}\right), i=1, \ldots, l+1$, and $h_{l+k} \in G^{\prime}, k=2, \ldots, t$.

The case $l=0$ was proved in Theorem 1. Suppose that $l \geq 1$.

For any $g \in G$ by $\bar{g}$ we denote its image in $A_{r}$. Then $\bar{h}_{i}=a_{i}, i=$ $1, \ldots, l+1 ; \bar{h}_{l+k}=1, k=2, \ldots, t$. Hence $\bar{H}=A_{l+1}=\left\langle a_{1}, \ldots, a_{l+1}\right\rangle$. By Baumslag's theorem [22] subgroup $H_{l+1}=\left\langle h_{1}, \ldots, h_{l+1}\right\rangle$ is isomorphic to $M_{l+1}$, and 
$\left\{h_{1}, \ldots, h_{l+1}\right\}$ is a basis of $H_{l+1}$. In particular, every map of this basis to $G$ extends to a homomorphism $H_{l+1} \rightarrow G$.

In the proof that follows we show that there is a homomorphism $\psi: G \rightarrow H$ identical on $H_{l+1}$, and then we show that it is identical on $H$, i.e., it is a retraction of $G$ onto $H$.

Let $h_{i}=h_{i}\left(z_{1}, \ldots, z_{r}\right), i=1, \ldots, l+1$ be expressions fixed generators of $H_{l+1}$ as words in basic elements of $G$. For any $i \in\{2, \ldots, l+1\}$ we consider equation

$$
\left[h_{i}\left(x_{1}, \ldots, x_{r}\right), h_{1}\left(x_{1}, \ldots, x_{r}\right), h_{1}\left(x_{1}, \ldots, x_{r}\right), h_{i}\left(x_{1}, \ldots, x_{r}\right)\right]=\left[h_{i}, h_{1}, h_{1}, h_{i}\right] .
$$

This equation is solvable in $G: x_{j}=z_{j}, j=1, \ldots, r$. Hence it has a solution in $H: x_{j}=f_{j}, f_{j} \in H, j=1, \ldots, r$.

We have:

$$
\begin{gathered}
{\left[h_{i}\left(f_{1}, \ldots, f_{r}\right), h_{1}\left(f_{1}, \ldots, f_{r}\right), h_{1}\left(f_{1}, \ldots, f_{r}\right), h_{i}\left(f_{1}, \ldots, f_{r}\right)\right] \equiv\left[h_{i}, h_{1}, h_{1}, h_{i}\right]\left(\bmod \gamma_{5} G\right),} \\
i=2, \ldots, l+1 .
\end{gathered}
$$

Denote $\tilde{h}_{j}=h_{j}\left(f_{1}, \ldots, f_{r}\right), j=1, i$. Let $\tilde{h}_{1} \equiv \prod_{j=1}^{l+1} z_{j}^{k_{j}}\left(\bmod G^{\prime}\right), \tilde{h}_{i} \equiv$ $\prod_{j=1}^{l+1} z_{j}^{m_{j}}\left(\bmod G^{\prime}\right), k_{j}, m_{j} \in \mathbb{Z}, j=1, \ldots, l+1$. Then (10) is equivalent to

$$
\left[\prod_{j=1}^{l+1} z_{j}^{m_{j}}, \prod_{j=1}^{l+1} z_{j}^{k_{j}}, \prod_{j=1}^{l+1} z_{j}^{k_{j}}, \prod_{j=1}^{l+1} z_{j}^{m_{j}}\right] \equiv\left[z_{i}, z_{1}, z_{1}, z_{i}\right]\left(\bmod \gamma_{5} G\right) .
$$

Quotient $G_{5}=G / \gamma_{5} G$ is a free group of variety $\mathfrak{A}^{2} \cap \mathfrak{N}_{5}$ of all metabelian nilpotent groups of nilpotency class $\leq 5$. Its the last non-trivial member of the low central series $\gamma_{4} G_{5}$ is a free abelian group with basis consisting of all basic commutators of length 4 of the form $\left[z_{i_{1}}, z_{i_{2}}, z_{i_{3}}, z_{i_{4}}\right]$, where $i_{1}>$ $i_{2}, i_{2} \leq i_{3} \leq i_{4}$. Any metabelian group satisfies to identities $\left[x_{1}, x_{2}, x_{3}, \ldots, x_{n}\right]=$ $\left[x_{1}, x_{2}, x_{\tau(3)}, \ldots, x_{\tau(n)}\right]$, where $n \geq 3, \tau$ is a substitution on $3, \ldots, n$. $G_{5}$ satisfies to identity $\left[x_{1}^{k_{1}}, x_{2}^{k_{2}}, x_{3}^{k_{3}}, x_{4}^{k_{4}}\right]=\left[x_{1}, x_{2}, x_{3}, x_{4}\right]^{k}, k=\prod_{j=1}^{4} k_{j}$. See details in [21].

These facts mean that after rewriting of the left side of (11) as a product of exponents of basic commutators of length 4 these exponents will be 0 with just one exception for exponent of $\left[z_{i}, z_{1}, z_{1}, z_{i}\right]$, which in correspondence with the right side of (11) should be 1 . We write a product of all basic commutators in this expression that depend of $z_{1}$ and $z_{i}$ only.

$$
\left[z_{i}, z_{1}, z_{1}, z_{i}\right]^{\alpha_{1}}\left[z_{i}, z_{1}, z_{1}, z_{1}\right]^{\alpha_{2}}\left[z_{i}, z_{1}, z_{i}, z_{i}\right]^{\alpha_{3}}
$$

where

$$
\alpha_{1}=\left(k_{1} m_{i}-k_{i} m_{1}\right)\left(k_{1} m_{i}+k_{i} m_{1}\right), \alpha_{2}=\left(k_{1} m_{i}-k_{i} m_{1}\right) k_{1} m_{1}, \alpha_{3}=\left(k_{1} m_{i}-k_{i} m_{1}\right) k_{i} m_{i} .
$$


A solution of (19) corresponds to values: $\alpha_{1}=1, \alpha_{2}=\alpha_{3}=0$. Note that $\delta=\left(k_{1} m_{i}-k_{i} m_{1}\right) \neq 0, \delta \in\{ \pm 1\}$, thus, $k_{1} m_{1}=k_{i} m_{i}=0$. Consider two the cases.

1) $k_{1}=0 \Rightarrow \delta=-k_{i} m_{1} \Rightarrow k_{i} \neq 0 \& m_{i}=0 \Rightarrow \alpha_{1}=-k_{i}^{2} m_{1}^{2}=1$, contradiction.

2) $m_{1}=0 \Rightarrow \delta=k_{1} m_{i} \neq 0 \Rightarrow m_{i} \neq 0 \& k_{i}=0 \Rightarrow \alpha_{1}=k_{1}^{2} m_{i}^{2}=1 \Rightarrow k_{1}=$ $\varepsilon_{1} \in\{ \pm 1\}, m_{i}=\varepsilon_{i} \in\{ \pm 1\}$, which gives a solution of (18).

Consider (10) again. It follows from our proof that the image of $\tilde{h}_{1}$ in $A_{r}$ does not depend of $a_{i}$, and the image of $\tilde{h}_{i}$ does not depend of $a_{1}$. Obviously these both the images as image of any element of $H$, does not depend of $a_{l+2}, \ldots, a_{r}$. The image of $\tilde{h}_{j}$ includes $a_{j}$ in exponent $\varepsilon_{j} \in\{ \pm 1\}, j=1, i$.

Consider a system of equations

$$
\begin{gathered}
{\left[h_{i}\left(x_{1}, \ldots, x_{r}\right), h_{1}\left(x_{1}, \ldots, x_{r}\right), h_{1}\left(x_{1}, \ldots, x_{r}\right), h_{i}\left(x_{1}, \ldots, x_{r}\right)\right]=\left[h_{i}, h_{1}, h_{1}, h_{i}\right],} \\
i=2, \ldots, l+1 .
\end{gathered}
$$

Here as above $h_{i}\left(x_{1}, \ldots, x_{r}\right)$ corresponds to $h_{i}=h_{i}\left(z_{1}, \ldots, z_{r}\right)$ that is an expression of $h_{i}$ by the basic elements of $G$.

Since $G$ is $l$-verbally closed this system has a solution in $G: x_{j}=z_{j}, j=$ $1, \ldots, r$. Hence it has a solution in $H: x_{j}=f_{j}, f_{j} \in H, j=1, \ldots, r$. Denote $u_{i}=h_{i}\left(f_{1}, \ldots, f_{r}\right), i=1, \ldots, l+1$. Let $\nu$ be an endomorphism of $G$ defined by the $\operatorname{map} \nu: z_{j} \mapsto f_{j}, j=1, \ldots, r$. Note that $u_{j}=\nu\left(h_{j}\left(z_{1}, \ldots, z_{r}\right)=\nu\left(h_{j}\right), j=1, \ldots, r\right.$. Then $\nu(G) \leq H$. The following equalities are valid:

$$
\left[u_{i}, u_{1}, u_{1}, u_{i}\right]=\left[h_{i}, h_{1}, h_{1}, h_{i}\right], i=1, \ldots, l+1 .
$$

In other words $\nu$ fixes every of elements $\left[h_{i}, h_{1}, h_{1}, h_{i}\right], i=1, \ldots, l+1$. The same is true for $\eta=\nu^{2}$. By arguments similar to ones about [?] applied to each of equations (20) we get congruence

$$
u_{1} \equiv h_{1}^{\varepsilon_{1}}\left(\bmod G^{\prime}\right), \varepsilon_{1} \in\{ \pm 1\} .
$$

By direct computation we obtain congruences $u_{i} \equiv h_{i}^{\varepsilon_{i}}\left(\bmod G^{\prime}\right), \varepsilon_{i} \in\{ \pm 1\}, i=$ $2, \ldots, l+1$. Let for example $u_{2} \equiv h_{2}^{\text {varepsilon }} h_{3}^{\lambda_{3}} \ldots h_{l+1}^{\lambda_{l+1}, \varepsilon_{2} \in\{ \pm 1}$ (this was proved above), $\lambda_{i} \in \mathbb{Z}$ and at least one of $\lambda_{j}$ is not 0 , let be $\lambda_{3}$. Then in expression of $\left[u_{2}, u_{1}, u_{1}, u_{2}\right]$ as a product of exponents of basic commutators modulo $\gamma_{5} \mathrm{H}$ of $\left[u_{2}, u_{1}, u_{1}, u_{2}\right]$ we have $\left[h_{3}, h_{1}, h_{1}, h_{3}\right]$ with exponent $\lambda_{3}^{2} \neq 0$, that contradics to (15).

Denote $w_{j}=\nu\left(u_{j}\right)=\eta\left(h_{j}\right)$, then $w_{i} \equiv z_{i}\left(\bmod G^{\prime}\right)$ and $w_{i} \equiv h_{i}\left(\bmod H^{\prime}\right), i=$ $1, \ldots, l+1$. We have a system of equalities

$$
\left[w_{i}, w_{1}, w_{1}, w_{i}\right]=\left[h_{i}, h_{1}, h_{1}, h_{i}\right], i=1, \ldots, l+1 .
$$


We use congruences $w_{i} \equiv h_{i}\left(\bmod H^{\prime}\right), i=1, \ldots, l+1$. Let $w_{i}=c_{i} h_{i}, c_{i} \in$ $H^{\prime}, i=1, \ldots, l+1$. Then

$$
\left[w_{i}, w_{1}, w_{1}, w_{i}\right]=\left[h_{i}, h_{1}, h_{1}, h_{i}\right] c_{i}^{\left(1-a_{1}\right)^{2}\left(1-a_{i}\right)} c_{1}^{\left(1-a_{i}\right)^{2}\left(a_{1}-1\right)}, i=2, \ldots, l+1 .
$$

After cancellations in exponents we obtain

$$
c_{1}^{1-a_{i}}=c_{i}^{1-a_{1}}, i=2, \ldots, l+1 .
$$

Hence there is $d \in H^{\prime}$ such that $c_{1}=d^{1-a_{1}}, c_{i}=d^{1-a_{i}}, i=2, \ldots, l+1$. The $\eta$ is inner automorphism corresponding to conjugation by $d \in H^{\prime}$. Define endomorphism $\psi$ as composition of $\eta$ with inner automorphism corresponding to conjugation by $d^{-1}$. Then $\psi$ maps $G$ to $H$ and is identical on $H_{l+1}$.

It remains to prove that $\psi$ is identical on $H$.

Let $h=h_{l+k}(k=2, \ldots, t)$ be one of the chosen generators of $H$. Then there is $0 \neq \alpha_{k} \in \mathbb{Z}\left[a_{1}^{ \pm 1}, \ldots, a_{l+1}^{ \pm 1}\right]$, such that $h^{\alpha_{k}} \in H_{l+1}$.

Let $h\left(z_{1}, \ldots, z_{r}\right)$ be an expression of $h$ as a word in basic elements of $G$. Then

$$
h\left(x_{1}, \ldots, x_{r}\right)=h
$$

is solvable in $G$. Hence it has a solution $f_{1}, \ldots, f_{r}$ in $H$. We substitute this solution to (20) and select in the left side $h_{l+j}, j=2, \ldots, t$. We obtain

$$
\tilde{h} \prod_{j=2}^{t} h_{l+j}^{\alpha_{k j}}=h, \tilde{h} \in H_{l+1}, \alpha_{k j} \in \mathbb{Z}\left[A_{l+1}\right]=\mathbb{Z}\left[a_{1}^{ \pm 1}, \ldots, a_{l+1}^{ \pm 1}\right] .
$$

We write this for every equation in (21) for $k=1, \ldots, t$, and obtain a system

$$
\tilde{h}_{l+k}=h_{l+k}^{1-\alpha_{k k}} \prod_{j=2, j \neq k}^{t} h_{l+j}^{-\alpha_{k j}}, \tilde{h}_{l+k} \in H_{l}, \alpha_{k j} \in \mathbb{Z}\left[A_{l}\right]=\mathbb{Z}\left[a_{1}^{ \pm 1}, \ldots, a_{l}^{ \pm 1}\right], k=2, \ldots, t .
$$

It remains to note that all $\alpha_{i j}$ in this equality lie in the fundamental ideal of the group ring $\mathbb{Z}\left[A_{l+1}\right]$, because $h_{l+k}\left(x_{1}, \ldots, x_{r}\right)$ is commutator word for every $k=2, \ldots, t$. Then $1-\alpha_{k k}$ is 1 modulo the fundamentsl ideal. It follows that determinant of the matrix consisting of exponent of the right side in (22) is not 0 . If consider $h_{l+k}, k=2, \ldots, t$, as unknowns the system is solvable over the field of fractions of the ring $\mathbb{Z}\left[A_{l+1}\right]$. Applying to (22) the Gauss elimination process we get

$$
h_{l+k}^{\alpha_{k}}=\tilde{h}_{k}, \alpha_{k} \in \mathbb{Z}\left[A_{l}\right], \tilde{h}_{k} \in H_{l+1}, k=2, \ldots, t .
$$

The endomorphism $\psi$ acts identically to $H_{l+1}$. Hence it induces identical map to $A_{l+1}$. Then

$$
\eta\left(h_{l+k}^{\alpha_{k}}\right)=\left(\eta\left(h_{l+k}\right)\right)^{\alpha_{k}}=h_{l+k}^{\alpha_{k}}, k=2, \ldots, t .
$$


It is known that $G^{\prime}$ has no module tersion. Since $\psi\left(h_{l+k}\right)=h_{l+k}$ for any $k=2, \ldots, t$, then $\psi$ is identical on $H$. This means that $\psi$ is a retraction $G \rightarrow H$.

\section{§3. PROBLEMS}

We put a few of natural problems.

Problem 1. Is it true that any finitely generated verbally closed subgroup of a free solvable group $S_{r, d}$ is a retract? What is the answer in a specific case $d=2$, i.e., in the case of a free metabelian group $M_{r}$ ?

More specific question:

Problem 2. Is it true that a subgroup $H$ of a free solvable group $S_{r, d}$ of class $d \geq 3$, such that $r_{a b}(H)=2$ is verbally closed if and only if $H$ is a retract of $S_{r, d}$ ?

A positive answer will strengthen Theorem 3 .

\section{References}

[1] W. Hodges, Model Theory, Cambridge, Cambridge Univ. Press, 1985.

[2] W.R. Scott, Algebraically closed groups, Proc. Amer. Math. Soc., 2 (1951), $118-121$.

[3] A. Myasnikov, V. Roman'kov, Verbally closed subgroups of free groups, J. Group Theory, 17, No. 1 (2014), 29-40.

[4] G. M. Bergman, Supports of derivations, free factorizations and ranks of fixed subgroups in free groups, Trans. Amer. Math. Soc., 351 (1999), 15511573.

[5] The Kourovka Notebook. Unsolved problems in group theory. No. 19 (2018),Editors: E.I. Khukhro and V.D. Mazurov. Sobolev Institute of Math. Siberian Branch RAS, Novosibirsk.

[6] G. Baumslag, A. Myasnikov, V. Shpilrain, Open problems in combinatorial group theory, Contemporary Math., 296 (2002), 1-38.

[7] I. Snopce, S. Tanushevski, P. Zalesskii, Retracts of free groups and a question of Bergman, arXiv:1902.02378 [math.GR]. Feb 6 (2019). 
[8] V.A. Roman'kov, N.G. Khisamiev, Verbally and existentially closed subgroups of free nilpotent groups. Algebra and Logic. 2013. 52, No. 4, 336-351.

[9] A. M. Mazhuga, On free decompositions of verbally closed subgroups in free products of finite groups, J. Group Theory, 20, No. 5 (2017), 971-986.

[10] A.M. Mazhuga, Strongly verbally closed groups, J. Algebra, 493 (2018), 171-184.

[11] A.M. Mazhuga, Verbally closed subgroups,Thesis, Lomonosov Moscow State University, Moscow, 2018 (In Russian).

[12] Ant. A. Klyachko, A.M. Mazhuga, Verbally closed virtually free subgroups, Sbornik: Mathematics, 209, No. 6 (2018), 850-856.

[13] A. A.Klyachko, A. M. Mazhuga, V. Y. Miroshnichenko, Virtually free finite-normal-subgroup-free groups are strongly verbally closed, J. Algebra, 510 (2018), 319-330.

[14] E.I. Timoshenko, Test elements and test rank of a free metabelian group, Siberian Mathematical Journal, 47, No. 6 (2000), 1200-1204.

[15] V.A. Roman'kov, Test elements for free solvable groups of rank 2. Algebra and Logic. 2001. 40, No.2. 106-111.

[16] E.I. Timoshenko, Computing test rank for a free solvable group. Algebra and Logic, 45,No. 4 (2006), 254-260.

[17] C.K. Gupta, E.I. Timoshenko, Test rank for fome free polynilpotent Groups, Algebra and Logic42, No. 1 (2003), 20-28.

[18] E.I. Timoshenko, Endomorphisms and universal theories of solvable groups. Novosibirsk State Technical University, Novosibirsk, 2011 (In Russian).

[19] V.N. Remeslennikov, V.A. Sokolov, Some properties of Magnus embedding, Algebra and Logic, 9 (1970), 342-349.

[20] R.H. Crowell, R.H. Fox, Introduction to knot theory, Springer-Verlag, Berlin-Heidelberg- New York, 1963.

[21] V. A. Roman'kov, Essays in algebra and cryptology. Solvable groups, Omsk, Omsk State University Publishing House, 2017. 
[22] G. Baumslag, Some subgroup theorems for free v-groups, Trans. Amer. Math. Soc., 108 (1963), 516-525.

[23] V.A. Roman'kov, Equations in free metabelian groups, Siberian Mathematical Journal. 1979. 20, No. 3 (1979). 469-471.

[24] H. Neumann, Varieties of groups, Springer-Verlag, Berlin-Heidelberg, 1967.

[25] V.A. Artamonov, Projective metabelian groups and Lie algebras, Uspekhi Mat. Nauk, 32, No. 3(195) (1977), 166.

[26] J. Lewin, A note on zero divisors in group rings, Proc. Amer. Math. Soc., 31 (1972), 357-359.

[27] C. K. Gupta, N.S. Romanovski, On torsion in factors of polynilpotent series of a group with a single relation, International Journal of Algebra and Computation, 14, No. 4 (2004), 513-523. 